\title{
Worsening Rural-Urban Gap in Hospital Mortality
}

\author{
Nicole Villapiano, MD, MSc, Theodore J. Iwashyna, MD, PhD, and \\ Matthew M. Davis, MD, MAPP
}

Background: One out of every 5 Americans live in rural communities. Rural Americans have higher rates of early and preventable deaths outside of the hospital than their urban counterparts. How rurality relates to hospital mortality is unknown. We sought to determine the association between rural versus urban residence and hospital mortality.

Methods: This is a retrospective observational study of 4,412,942 nonmaternal, nonneonatal hospitalizations in 2008, and 3899,464 nonmaternal, nonneonatal hospitalizations in 2013 using all-payer, all-age data from the National Inpatient Sample of the Health care Cost and Utilization Project. Using multivariable logistic regression, we report the association between rural versus urban location of residence and hospital mortality, adjusting for chronic disease burden, age, income, and insurance status.

Results: The unadjusted probability of hospital mortality for urban patients decreased from $2.51 \%$ (95\% CI, 2.40 to 2.62$)$ in 2008 to $2.27 \%(95 \%$ CI, 2.22 to 2.32$)$ in $2013(P<.001)$. Hospital mortality did not change for rural patients over this same time period (2008: 2.66\% [95\% CI, 2.57 to 2.74], 2013: $2.66 \%$ [95\% CI, 2.60 to 2.72]; $P=.99)$. Adjusting for covariates accounted for the rural-urban hospital mortality difference in 2008 (rural: $2.13 \%$ [95\% CI, 2.05 to 2.21], urban: $2.11 \%$ [95\% CI, 2.02 to 2.20]; $P=.67$ ), but did not fully explain the difference in 2013 (rural: $1.92 \%$ [95\% CI, 1.87 to 1.97]; urban: $1.76 \%$ [ $95 \%$ CI, 1.72 to 1.80 ], $P<.001$ ), resulting in 8416 excess deaths among hospitalized patients from rural areas.

Conclusion and Relevance: In 2013, patients living in rural areas of the United States had a greater probability of hospital mortality than their urban counterparts. Explaining excess rural hospital deaths will require further attention to the patient, community, and health system factors that distinguish rural from urban populations. (J Am Board Fam Med 2017;30:816-823.)

Keywords: Health Care Disparities, Hospital Mortality, Hospitalists, Rural Health

Nearly 1 in 5 Americans live in rural areas. ${ }^{1} \mathrm{~Pa}$ tients living in rural areas have higher chronic disease burden, lower incomes, and less access to

This article was externally peer reviewed.

Submitted 23 March 2017; revised 25 July 2017; accepted 1 August 2017.

From the Robert Wood Johnson Foundation Clinical Scholars Program, Institute for Healthcare Policy and Innovation, Department of Internal Medicine, University of Michigan, Ann Arbor, MI (NV); VA Center for Clinical Management Research, Ann Arbor and Institute for Healthcare Policy and Innovation, Department of Internal Medicine, University of Michigan, Ann Arbor (TJI); Ann \& Robert H. Lurie Children's Hospital, Division of Academic General Pediatrics, Mary Ann and J. Milburn Smith Child Health Research Program, Departments of Pediatrics and Medical Social Sciences, Northwestern University Feinberg School of Medicine, Chicago, IL (MMD).

Funding: This publication was supported by the Robert Wood Johnson Foundation Clinical Scholars Program. The funder had no role in the study design, data collection, analysis, interpretation of data, manuscript review, or decision to submit the manuscript for publication. health care than their urban counterparts. ${ }^{2,3}$ Moreover, the aging rural population faces social, economic, and geographic barriers to health that have created widening rural-urban disparities in life expectancy, premature mortality, and infant mortality. 3,4

Yet, whereas these population-health facts are known, far less is known about modern rural hospital care relative to care delivered in the urban setting. Recent studies of critical access hospitals, which account for $68 \%$ of rural hospitals ${ }^{5}$, offer conflicting pictures of quality and outcomes. Joynt ${ }^{6}$

Conflict of interest: none declared.

Disclaimer: This work does not represent the views of the US Government or Department of Veterans Affairs.

Corresponding author: Nicole Villapiano, MD, MSc, University of Michigan, Robert Wood Johnson Foundation Clinical Scholars Program, North Campus Research Center, 2800 Plymouth Road, Building 14 G100-36, Ann Arbor, MI 48109-2800 (E-mail: gergen@med.umich.edu). 
and colleagues described greater hospital mortality for acute myocardial infarction, congestive heart failure, and pneumonia among Medicare patients hospitalized at critical access hospitals, compared with Medicare patients hospitalized at predominantly urban noncritical access hospitals. Furthermore, between 2002 and 2010 overall hospital mortality increased for Medicare patients admitted to critical access hospitals, whereas mortality rates for Medicare patients in noncritical access hospitals improved during this same time period. ${ }^{6}$ In contrast, Ibrahim et $\mathrm{al}^{7}$ showed that Medicare patients admitted to critical access hospitals for common surgical procedures had equivalent mortality to Medicare patients admitted to noncritical access hospitals.

Given the large population that lives in rural areas and their ongoing demographic changes, rare all-payer assessments of the hospitalized rural population, emerging value-based hospital reimbursement arrangements, and changes in health care systems driving tighter alignment between primary care and hospital incentives, we sought to provide the first comprehensive study of hospital mortality among rural Americans compared with urban Americans. Using cross-sectional, nationally representative samples of inpatients across all payers, we measured hospital mortality for rural versus urban hospitalized patients over the most recently available 5 -year period (2008 to 2013). We then examined the relationship between hospital mortality, patient demographics, and chronic disease burden over this time period, and described rural-urban differences in hospital mortality for common conditions. The relationship between rurality and hospital mortality, and potential differences compared with urban populations could prompt health systems, communities, public health officials, and policy makers to identify opportunities for future quality improvement efforts that narrow identified gaps.

\section{Methods}

\section{Data Source}

We utilized data from the National Inpatient Sample (NIS) developed by the Agency for Health care Research and Quality's Health care Cost and Utilization Project (HCUP). ${ }^{8}$ The NIS is the largest all-payer annual database of hospital discharges in the United States, and includes patient and hospital-level data from more than 1000 nonfederal hospitals in 46 states, encompassing $97 \%$ of the US population and $94 \%$ of all hospital discharges. To account for sampling redesign in 2012, trend weights provided by HCUP were applied to 2008 data to allow for comparison with data from 2013.

\section{Study Population}

For the years 2008 and 2013, we evaluated all nonmaternal, nonneonatal hospitalizations for patients living in rural or urban areas of the United States.

\section{Predictor and Outcome Variables}

The interaction between patient location of residence and study year was used to estimate primary outcomes related to rural versus urban differences. Rural-urban status was determined in the NIS using the National Center for Health Statistics Classification Scheme for Counties based on Metropolitan Statistical Areas. ${ }^{9}$ For purposes of our analysis, urban was defined as counties designated as either central or fringe large metro areas with a population of more than 1 million people. Rural included both micropolitan counties (centered around a city of 10,000 to 50,000 people) and noncore counties. Suburban counties were not included in this analysis. The primary outcome of this study was hospital mortality, defined as death in the hospital before discharge.

\section{Analysis}

We analyzed the relationship between the interaction of patient location of residence with year and hospital mortality using multivariable logistic regression, applying sampling weights to all analyses to permit national inferences. We present the unadjusted model for comparison with models adjusted for the individual effect of chronic disease burden as measured by the number of Elixhauser comorbidities (a comprehensive set of 30 comorbidity measures that are commonly used to predict risk of inpatient mortality) attributed to the patient, and sociodemographic factors including age, sex, income, and payer (Medicare, Medicaid, private insurance, or uninsured). ${ }^{10}$ The final model measured hospital mortality as predicted by rural-urban status and year, adjusted for all covariates.

Next, we calculated marginal adjusted predicted probabilities of hospital death for rural and urban hospitalized patients in 2008 and 2013. The probability difference between rural and urban hospital 
mortality was used to estimate excess deaths for each year.

We then determined which 15 primary diagnoses most frequently resulted in hospital death. Clinical Classification Software developed by the Agency for Health care Research and Quality was used to aggregate the International Classification of Diseases-Ninth Revision (ICD-9) code associated with each primary diagnosis observation in the dataset into 1 of 271 unique, clinically relevant diagnoses. Unadjusted logistic regression was performed to determine the odds of death in the hospital for the most commonly fatal diagnoses (abdominal aortic aneurysm, sepsis, respiratory failure, gastrointestinal bleed, acute myocardial infarction, secondary malignancy, pneumonia, chronic obstructive pulmonary disease, aspiration pneumonia, stroke, congestive heart failure, renal failure, intracranial hemorrhage, lung cancer, and bowel obstruction) for both rural and urban patients in 2013.

Another logistic regression model was developed to predict the probability of hospital mortality by the interaction of rural-urban status, payer, and year while controlling for age, sex, chronic disease burden, and income to measure hospital mortality within payer subgroups (Medicare, Medicaid, privately insured, and uninsured). Predicted probabilities of hospital death with $95 \%$ CIs were calculated at each year for hospitalized rural and urban patients. Excess hospital mortality per 1000 hospitalized patients by payer subgroup was calculated using the probability difference between rural and urban hospital mortality in both 2008 and 2013. Two-sided $P$ values less than .05 were considered statistically significant differences.

All analyses were performed using STATA software, version 14 (StataCorp, College Station, TX). The institutional review board of the University of Michigan Medical School deemed this study of publicly available, deidentified data exempt from human subjects review.

\section{Results}

There were 8312,406 nonmaternal nonneonatal hospital admissions and 206,020 hospital deaths among rural and urban patients included in the study sample, accounting for over 40 million weighted hospitalizations and over 957,000 weighted hospital deaths. Hospitalized rural patients were older and more likely be insured by Medicare in both 2008 and 2013. Al- though chronic disease burden increased between 2008 and 2013 for both rural and urban hospitalized patients, in 2013 a greater percentage of rural patients had 5 or more chronic diseases (rural, $59.1 \%$ vs urban, 56.4\%). A large income disparity existed between rural and urban hospitalized patients. In both 2008 and 2013 , over $86 \%$ of rural hospitalized patients had incomes in the lower half of the income distribution, whereas less than less than $43 \%$ of urban patients were among these low-income groups. (Table 1).

The unadjusted probability of hospital mortality for urban hospitalized patients decreased from $2.51 \%$ (95\% CI, 2.40 to 2.62 ) in 2008 to $2.27 \%$ (95\% CI, 2.22 to 2.32$)$ in $2013(P<.001)$. In contrast, hospital mortality did not improve for rural patients over this same time period (unadjusted probability of hospital mortality, 2008: $2.66 \%$ [95\% CI, 2.57 to 2.74]; 2013: $2.66 \%$ [95\% CI, 2.60 to 2.72 ]; $P=.99$; Table 2).

In 2008, adjustment for patient comorbidity, age, sex, income, and payer status explained the unadjusted rural-urban hospital mortality difference (adjusted probability of hospital mortality, rural: 2.13 [95\% CI, 2.05 to 2.21 ]; urban: $2.11 \%$ [95\% CI, 2.02 to 2.20]; $P=.67$ ), whereas in 2013, this adjustment did not fully explain the mortality difference (adjusted probability of hospital mortality, rural: $1.92 \%$ [95\% CI, 1.87 to 1.97 ]; urban: $1.76 \%$ [95\% CI, 1.72 to 1.80 ]; $P<.001$; Table 2). The differences in adjusted probabilities of hospital mortality for rural versus urban patients in 2013 corresponded to 8416 excess deaths among hospitalized patients from rural areas.

The magnitude of the rural versus urban difference in hospital mortality in 2013 varied by primary diagnosis. Rural patients were more likely than urban patients to die in the hospital for over half $(8 / 15)$ of the diagnoses most frequently identified in cases of hospital mortality (Figure 1). The odds of death in the hospital for rural and urban patients were not different for 6 of these diagnoses. Sepsis was the only of the most common fatal conditions more likely to result in hospital death for urban patients versus rural patients (Figure 1). Rural patients with lung cancer, renal failure, and intracranial hemorrhage had the greatest odds of death in the hospital compared with urban patients with the same primary diagnoses.

After adjusting for covariates, the magnitude of the rural-urban differences in hospital mortality 
Table 1. Demographic Characteristics of Patients Admitted to the Hospital by Rural or Urban Residence, 2008 and 2013

\begin{tabular}{|c|c|c|c|c|c|c|}
\hline & \multicolumn{2}{|c|}{2008} & \multirow[b]{2}{*}{$P$ Value } & \multicolumn{2}{|c|}{2013} & \multirow[b]{2}{*}{$P$ Value } \\
\hline & Urban (\%) & Rural (\%) & & Urban (\%) & Rural (\%) & \\
\hline Unweighted discharges (n) & $3,206,376(52.0)$ & $1,206,566(19.8)$ & $<.001$ & $2,870,054(52.4)$ & $1029,410(18.8)$ & $<.001$ \\
\hline Weighted discharges $(\mathrm{N})$ & $15,795,128(53.1)$ & $5,916,858(19.9)$ & $<.001$ & $14,350,107(52.4)$ & $5,146,547(18.8)$ & $<.001$ \\
\hline Age (years) & & & $<.001$ & & & $<.001$ \\
\hline 0 to 17 & 5.4 & 5.5 & & 6.1 & 4.8 & \\
\hline 18 to 35 & 9.9 & 8.0 & & 10.4 & 7.9 & \\
\hline 36 to 50 & 17.6 & 14.7 & & 15.3 & 13.1 & \\
\hline 51 to 64 & 22.9 & 22.2 & & 24.6 & 24.1 & \\
\hline 65 to 74 & 16.3 & 19.5 & & 17.6 & 21.2 & \\
\hline $75+$ & 27.9 & 30.1 & & 26.1 & 29.0 & \\
\hline Sex & & & .674 & & & .505 \\
\hline Female & 46.3 & 53.9 & & 52.4 & 52.4 & \\
\hline Male & 53.7 & 46.1 & & 47.6 & 47.6 & \\
\hline Payer & & & $<.001$ & & & $<.001$ \\
\hline Medicare & 46.3 & 54.1 & & 48.3 & 56.9 & \\
\hline Medicaid & 12.1 & 10.9 & & 15.0 & 11.6 & \\
\hline Private & 32.8 & 26.4 & & 26.8 & 22.1 & \\
\hline Uninsured & 8.8 & 8.7 & & 10.0 & 9.4 & \\
\hline Number of chronic conditions & & & $<.626$ & & & $<.001$ \\
\hline 0 & 6.2 & 6.4 & & 5.0 & 4.3 & \\
\hline 1 & 9.5 & 9.0 & & 7.5 & 6.4 & \\
\hline 2 & 24.6 & 11.3 & & 9.0 & 8.2 & \\
\hline 3 to 4 & 13.7 & 27.1 & & 22.1 & 21.9 & \\
\hline $5+$ & 46.1 & 46.0 & & 56.4 & 59.1 & \\
\hline Length of stay (days) & & & $<.001$ & & & $<.001$ \\
\hline 0 to 2 & 36.8 & 37.7 & & 36.5 & 35.8 & \\
\hline 3 to 4 & 35.3 & 37.4 & & 36.5 & 38.9 & \\
\hline 5 to 7 & 10.3 & 10.1 & & 10.3 & 10.3 & \\
\hline 8 to 14 & 12.0 & 10.7 & & 11.7 & 10.9 & \\
\hline$>14$ days & 5.7 & 4.2 & & 5.0 & 4.1 & \\
\hline Income quartile & & & $<.001$ & & & $<.001$ \\
\hline 1 (lowest income) & 20.5 & 52.1 & & 22.2 & 49.5 & \\
\hline 2 & 21.0 & 36.8 & & 20.4 & 37.4 & \\
\hline 3 & 26.4 & 9.6 & & 26.4 & 11.6 & \\
\hline 4 (highest income) & 32.1 & 1.5 & & 31.0 & 1.5 & \\
\hline
\end{tabular}

varied by payer (Figure 2). No rural-urban differences in hospital mortality were observed across payer subgroups in 2008; however, in 2013 hospital mortality for rural patients was higher among those with Medicare, Medicaid, and private insurance. The greatest rural-urban difference occurred among Medicaid patients in 2013 (adjusted probability of hospital mortality, rural: $2.47 \%$ [95\% CI, 2.32 to 2.62 ]; urban: $2.08 \%$ [95\% CI, 2.00 to 2.16 ]; $P<.001$ ), resulting in nearly 4 excess deaths among hospitalized Medicaid patients living in rural areas for every 1000 patients admitted to the hospital. Uninsured patients had the highest probabilities of hospital mortality in the years studied, but there were no differences across years or by rural-urban status (adjusted probability of hospital mortality, 2008: rural, 3.07\% [95\% CI, 2.82 to 3.47]; urban, 3.57\% [95\% CI, 2.88 to 4.26]; 2013: rural, $2.84 \%$ [95\% CI, 2.65 to 3.05 ]; urban, $2.87 \%$ [95\% CI, 2.65 to 3.10]).

\section{Discussion}

In 2008, differences in hospital mortality for rural versus urban patients were not observed, but in 2013 , the probability of rural patients dying in the 
Table 2. Unadjusted and Adjusted Models Predicting the Probability of Hospital Mortality for Urban Versus Rural Patients, 2008 and 2013

\begin{tabular}{|c|c|c|c|c|c|c|c|c|c|c|}
\hline \multirow[b]{2}{*}{ Model } & \multicolumn{5}{|c|}{2008} & \multicolumn{5}{|c|}{2013} \\
\hline & Urban & $(95 \% \mathrm{CI})$ & Rural & $(95 \% \mathrm{CI})$ & $P$ Value & Urban & $(95 \% \mathrm{CI})$ & Rural & $(95 \% \mathrm{CI})$ & $P$ Value \\
\hline Unadjusted & $2.51 \%$ & (2.40 to 2.62 ) & $2.66 \%$ & (2.57 to 2.74$)$ & $<.05$ & $2.27 \%$ & (2.22 to 2.32$)$ & $2.66 \%$ & (2.60 to 2.71$)$ & $<.001$ \\
\hline $\begin{array}{l}\text { Adjusted for chronic } \\
\text { disease burden }\end{array}$ & $2.30 \%$ & (2.24 to 2.44$)$ & $2.60 \%$ & (2.51 to 2.66$)$ & $<.001$ & $1.80 \%$ & (1.79 to 1.87$)$ & $2.20 \%$ & (2.14 to 2.24$)$ & $<.001$ \\
\hline Adjusted for age & $2.04 \%$ & (2.13 to 1.88$)$ & $2.05 \%$ & (1.97 to 2.13$)$ & .913 & $1.88 \%$ & (1.84 to 1.92$)$ & $2.06 \%$ & (2.00 to 2.11 ) & $<.001$ \\
\hline Adjusted for sex & $2.51 \%$ & (2.40 to 2.62 ) & $2.66 \%$ & (2.57 to 2.74$)$ & $<.05$ & $2.27 \%$ & (2.22 to 2.32$)$ & $2.66 \%$ & (2.60 to 2.71$)$ & $<.001$ \\
\hline Adjusted for income & $2.50 \%$ & (2.39 to 2.61 ) & $2.67 \%$ & (2.59 to 2.79$)$ & $<.01$ & $2.26 \%$ & (2.21 to 2.32 ) & $2.68 \%$ & (2.61 to 2.74 ) & $<.001$ \\
\hline Adjusted for payer & $2.35 \%$ & (2.25 to 2.45$)$ & $2.34 \%$ & (2.26 to 2.42$)$ & .863 & $2.09 \%$ & (2.05 to 2.14$)$ & $2.29 \%$ & (2.23 to 2.34$)$ & $<.001$ \\
\hline $\begin{array}{l}\text { Adjusted for chronic } \\
\text { disease, age, sex, } \\
\text { income, payer }\end{array}$ & $2.11 \%$ & (2.02 to 2.20$)$ & $2.13 \%$ & (2.05 to 2.21$)$ & .665 & $1.76 \%$ & (1.72 to 1.80$)$ & $1.92 \%$ & (1.87 to 1.97$)$ & $<.001$ \\
\hline
\end{tabular}

$P$ values reported measure the significance of the urban-rural comparison in 2008 and 2013.

CI, confidence interval.

hospital was nearly $10 \%$ greater than that of their urban counterparts. This newly identified gap in adjusted hospital mortality resulted in over 8000 excess deaths among rural hospitalized patients in 2013. No single diagnosed condition accounts for the rural versus urban hospital mortality gap in 2013, however, rural Medicaid patients had the highest probability of death in the hospital.

Our findings suggest the presence of unmeasured factors related to rurality that have progres- sively contributed to hospital mortality in recent years. From a population-health perspective, the relative extent to which patient, community, and/or health system factors may be attributable to the rural-urban hospital mortality gap is unknown and merits further attention.

Rural patients are known to have higher levels of health-risk-taking behaviors such as smoking and less healthy diets than their urban counterparts, which may not be fully accounted for by chronic

Figure 1. Hospital mortality by condition in 2013, odds ratios (95\% CIs). CI, confidence interval; COPD, Chronic obstructive pulmonary disease.

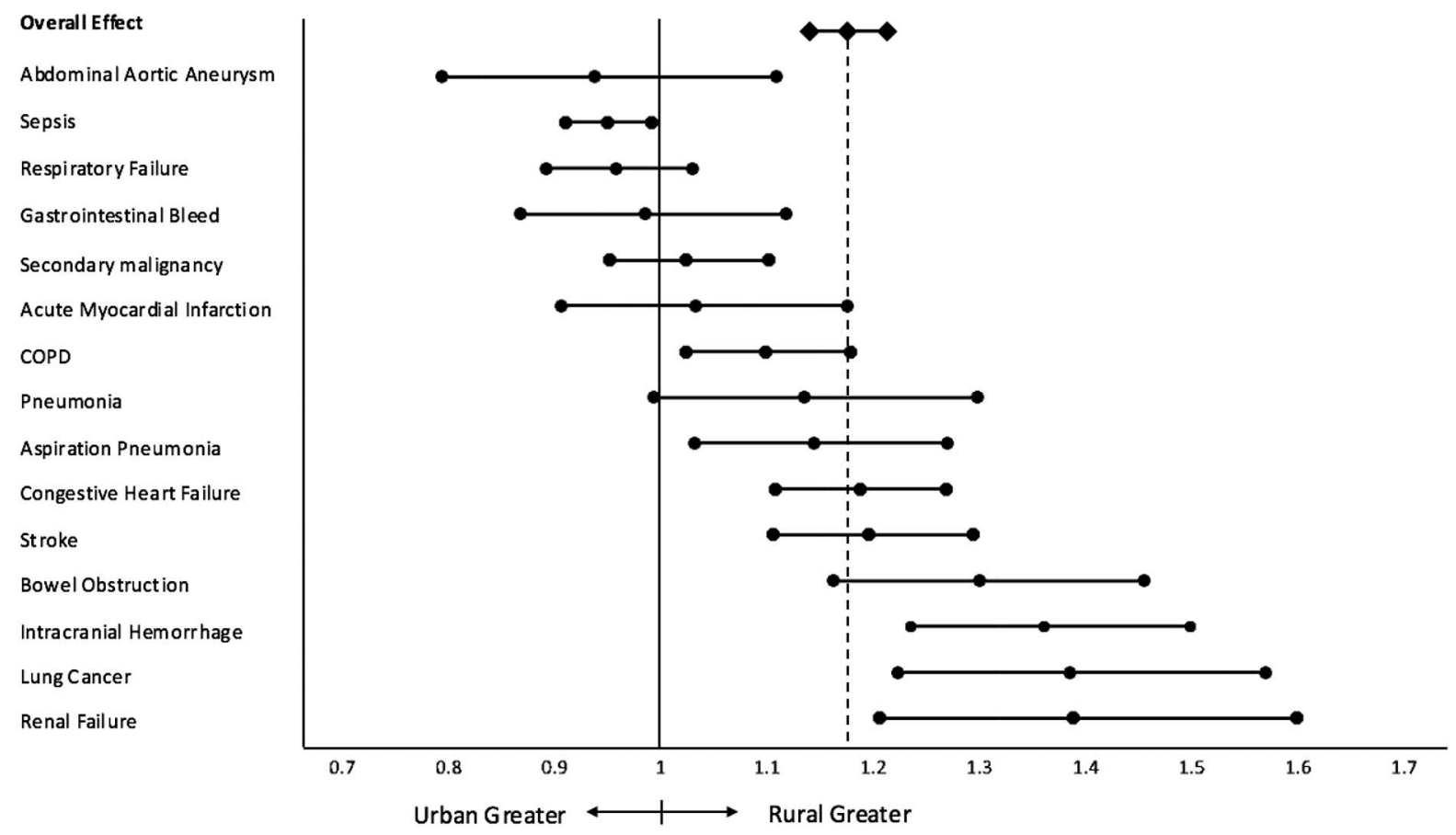


Figure 2. Excess hospital deaths among rural patients per 1000 patients hospitalized, by year and payer status. Data as measured from a multivariable model including patient age, sex, and income, and Elixhauser chronic disease burden index. *Excess hospital mortality significant at $P<.05$.

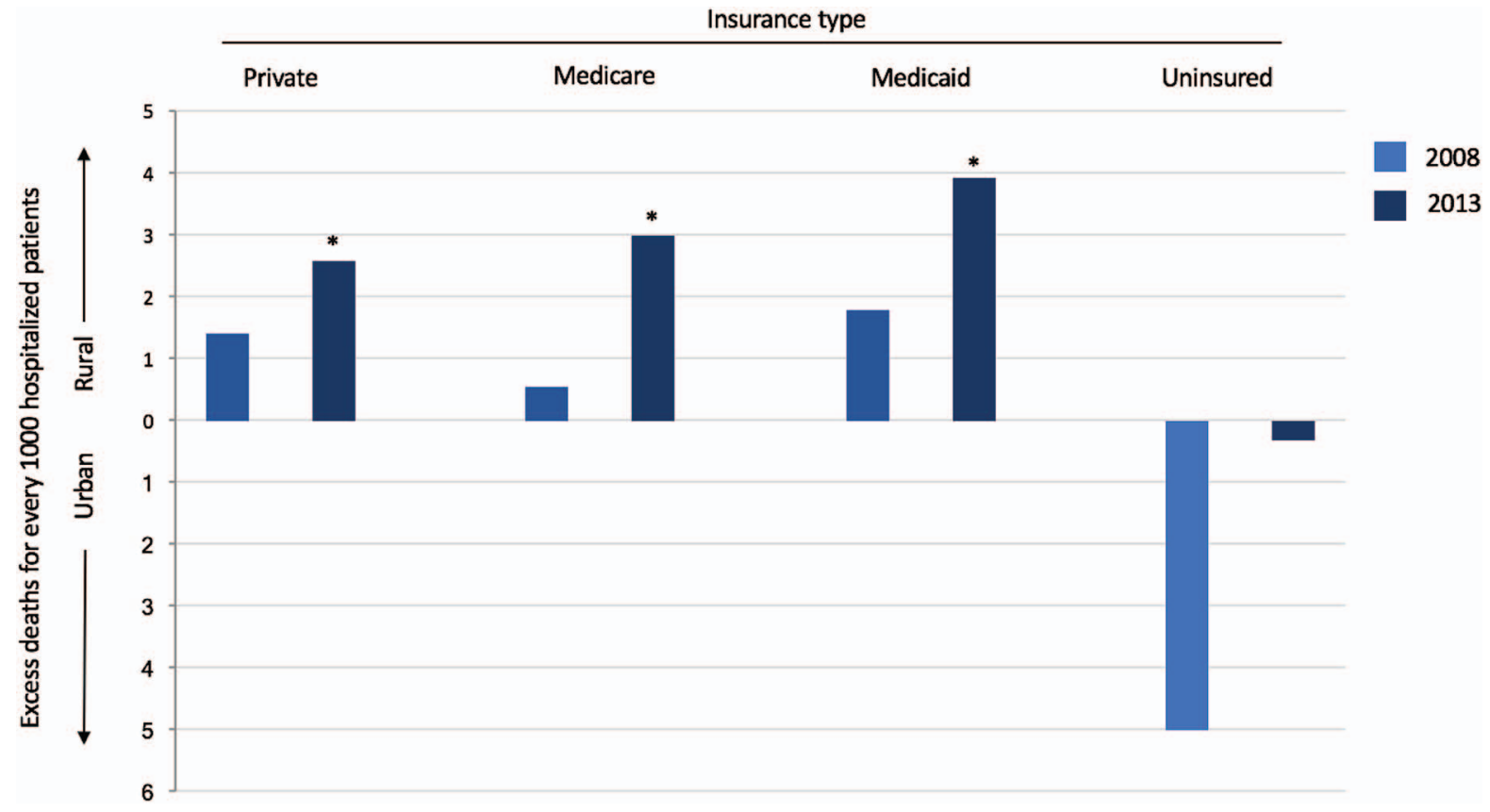

disease indices used to analyze claims data, and may contribute to higher rates of overall hospital mortality. ${ }^{2,11-13}$ There is also concern that community-level factors including living environment, community context, distance to providers, and local economic prospects could influence hospital mortality. ${ }^{14-16}$ Community factors such as the number of primary care doctors per capita, and the average proportion of high-risk, long-stay nursing home patients have been demonstrated to explain over half of the variability in hospital readmission rates between counties, but it is not clear how these factors relate to recent trends in hospital mortality. ${ }^{17}$

Within health systems, several other factors may be contributing to the rural-urban hospital mortality gap. At the hospital level, it is possible that hospitals serving rural patients are not providing care of improved quality, and are not contributing to improving outcomes over time. Previous studies, however, suggest mixed results regarding this possibility. ${ }^{6,7}$ Due to limitations of claims data, these studies cannot address the nuanced calculation of hospital mortality in the context of the mortality rate of the local nonhospitalized population, or the unique challenges of risk-adjusting quality measurement in rural areas. ${ }^{18,19}$ In addition, previous reports of lower rural and community hospital quality have not considered patient preferences, triage decision trees, or admission thresholds. ${ }^{20-22}$ Such factors may predispose rural hospitals to transfer younger, healthier patients with treatable conditions to urban tertiary centers while admitting older, sicker patients with higher disease-specific risk of mortality; the latter group may include patients who either may not stand to benefit from the services offered at the tertiary center or who may not wish to seek advanced care for their conditions. ${ }^{23}$ Our data reporting the greatest probability of hospital death among rural patients admitted with intracranial hemorrhage, renal failure, and lung cancer may support this theory, given that these 3 diagnoses are frequently incurable and may be associated with goals of care that do not involve advanced treatments. This in combination with restricted access to hospice services in rural areas may predispose more rural residents to die in the hospital with untreatable diseases rather than in outpatient facilities. ${ }^{24-30}$

National data also suggests a growing trend toward rural residents bypassing rural hospitals and receiving care at urban hospitals. ${ }^{31}$ Rural patients hospitalized in urban centers are more likely to be younger, male, and not insured by Medicare. They 
additionally are more likely to have procedures performed during their hospitalizations. ${ }^{31}$ It is not clear from our data how bypass patterns may be effecting hospital mortality trends for rural patients. The trend toward rural hospital bypass in combination with the newly emerging hospital mortality gap among rural patients could suggest that rural hospital quality may not be driving the rural-urban hospital mortality gap. Previous work has shown that the increasing trend toward rural hospital bypass may be putting patients with timesensitive conditions at greater risk of hospital death due to delays in care related to transportation. ${ }^{32}$ Alternatively, this could suggest lower quality of care at urban hospitals. Future work is needed to fully evaluate these hypotheses.

Finally, within health systems, we must consider the impact of availability of local primary care, specialist, rehabilitation, and ancillary services on driving hospitalization decisions and ultimately hospital mortality outcomes. For example, communities with health systems that support a robust hospice program have demonstrated reduced hospital mortality rates. ${ }^{33}$ Our data source did not permit consideration of patient, community, or health system factors in our analyses; rather, our findings suggest that these factors warrant more investigation.

To disentangle the factors explaining the emerging hospital mortality gap for rural versus urban patients, future work exploring hospital mortality differences should take a population health approach-prospectively collecting data on community factors, patient demographics, patient preferences, hospital characteristics, and place to death to better address the role hospital mortality plays in population health for rural communities.

\section{Limitations}

The implications of our analyses must be understood in light of specific analytic limitations. Our study could not account for behavioral and lifestyle factors, such as smoking and dietary habits, which may adversely affect the chronic disease burden and hospital mortality differences observed for rural versus urban patients. In addition, we did not have data on primary care utilization to assess the impact of access to outpatient medical care. Hospital critical access status, and other hospital-level factorssuch as nurse-patient staffing ratios, availability of medical technology, and workforce training-that may influence delivery of appropriate care, were not available from our data source and therefore were not accounted for in this study. Finally, this study did not account for rural versus urban hospital categorization, which is a potentially important factor in identifying the underlying causes of the hospital mortality gap observed in 2013.

\section{Conclusions}

In rural areas of the United States, where nearly 1 out of every 5 Americans live, patients experienced significantly higher hospital mortality than their urban counterparts in 2013. This disparity is only partially explained by observable differences in the demographic and socioeconomic realities of rural versus urban populations in the United States today. To address excess rural deaths in health care settings will require specific attention to evolving patient, community, and health system factors that may differ in predictable and preventable ways for rural versus urban groups.

To see this article online, please go to: http://jabfm.org/content/ 30/6/816.full.

\section{References}

1. Ratcliffe M, Burd C, Holder K, Fields A. Defining rural at the U.S. Census Bureau. ACSGEO-1. Washington, DC: U.S. Census Bureau, 2016.

2. Singh GK, Siahpush M. Widening rural-urban disparities in life expectancy. U.S., 1969-2009. Am J Prevent Med 2014;46(2):e19-e29.

3. Singh GK, Siahpush M. Widening rural-urban disparities in all-cause mortality and mortality from major causes of death in the USA, 1969-2009. J Urban Health 2014;91:272-292.

4. Moy E, Garcia MC, Bastian B, et al. Leading causes of death in nonmetropolitan and metropolitan areas-United States, 1999-2014. MMWR Surveill Summ 2017;66:1-8.

5. Center for Medicare and Medicaid Services. Critical access hospitals. Department of Health and Human Services Center for Medicare and Medicaid Services Medicare Learning Network. February 2016. Available from: https://www.cms.gov/Outreach-andEducation/Medicare-Learning-Network-MLN/MLN Products/downloads/CritAccessHospfctsht.pdf. Accessed January 18, 2017.

6. Joynt KE, Orav EJ, Jha AK. Mortality rates for Medicare beneficiaries admitted to critical access and non-critical access hospitals, 2002-2010. JAMA 2013;309:1379-1387.

7. Ibrahim AM, Hughes TG, Thumma JR, Dimick JB. Association of hospital critical access status with sur- 
gical outcomes and expenditures among Medicare beneficiaries. JAMA 2016;315:2095-2103.

8. Healthcare Cost and Utilization Project (HCUP) databases. Agency for Healthcare Research and Quality. Available from: http://www.hcup-us.ahrq. gov/databases.jsp. Accessed January 18, 2017.

9. Ingram DD, Franco SJ. 2013 NCHS urban-rural classification scheme for counties. Vital Health Stat 2014;2:1-73.

10. Elixhauser A, Steiner C, Harris DR, Coffey RM. Comorbidity measures for use with administrative data. Med Care 1998;36:8-27.

11. Logan H, Guo Y, Dodd VJ, Muller K, Riley J 3rd. The burden of chronic diseases in a rural North Florida sample. BMC Public Health 2013;13:906.

12. Gamm L, Hutchison L, Bellamy G, Dabney BJ. Rural healthy people 2010: Identifying rural health priorities and models for practice. J Rural Health 2002;18:9-14.

13. Smalley KB, Warren JC, Klibert J. Health risk behaviors in insured and uninsured community health center patients in the rural US South. Rural and remote health 2012;12:2123.

14. Henning-Smith C, Kozhimannil K, Prasad S, Casey M, Moscovice I. Quality measures and sociodemographic risk factors: The Rural Context. University of Minnesota Rural Health Research Center Policy Brief. May 2016. Available from: http://rhrc. umn.edu/wp-content/uploads/2016/05/QualityMeasures-and-Sociodemographic-Risk-Factors.pdf. Accessed January 18, 2017.

15. Moist LM, Bragg-Gresham JL, Pisoni RL, et al. Travel time to dialysis as a predictor of health-related quality of life, adherence, and mortality: The Dialysis Outcomes and Practice Patterns Study (DOPPS). Am J Kidney Dis. 2008;51:641-650.

16. Nicholl J, West J, Goodacre S, Turner J. The relationship between distance to hospital and patient mortality in emergencies: An observational study. EMJ 2007;24:665-668.

17. Herrin J, St Andre J, Kenward K, Joshi MS, Audet AM, Hines SC. Community factors and hospital readmission rates. Health Serv Res 2015;50: 20-39.

18. McCormick B, Pearson M, White J. Hospital mortality rates and place of death. J Pub Health (Oxf). 2016;38:800-805.

19. Moscovice IS, Casey MM, McCullough JS. Mortality trends in critical access hospitals. JAMA 2013; 310:428-429.

20. Pitches DW, Mohammed MA, Lilford RJ. What is the empirical evidence that hospitals with higher-risk adjusted mortality rates provide poorer quality care?
A systematic review of the literature. BMC Health Serv Res 2007;7:91.

21. Lilford R, Pronovost P. Using hospital mortality rates to judge hospital performance: A bad idea that just won't go away. BMJ 2010;340:c2016.

22. Finlayson SR, Birkmeyer JD, Tosteson AN, Nease RF Jr. Patient preferences for location of care: Implications for regionalization. Med Care 1999;37: 204-209.

23. Mehta RH, Stalhandske EJ, McCargar PA, Ruane TJ, Eagle KA. Elderly patients at highest risk with acute myocardial infarction are more frequently transferred from community hospitals to tertiary centers: Reality or myth? Am Heart J 1999;138(4 Pt 1):688-695.

24. National Advisory Committee on Rural Health and Human Services. Rural implications of changes to the medicare hospice benefit. 2013. Available from: https://www.hrsa.gov/advisorycommittees/rural/ publications/nacrhhshospicebrief.pdf. Accessed July 13, 2017.

25. Radcliff TA, Brasure M, Moscovice IS, Stensland JT. Understanding rural hospital bypass behavior. J Rural Health 2003;19:252-259.

26. Goldsteen RL, Falcone DJ, Broyles RW, Goldsteen $\mathrm{K}$, Reilly BJ. Local factors affecting the tendency to bypass local hospitals for inpatient mental health care: An exploratory analysis. J Rural Health 1994; 10:89-97.

27. Adams EK, Wright GE. Hospital choice of Medicare beneficiaries in a rural market: Why not the closest? J Rural Health 1991;7(2):134-52.

28. Escarce JJ, Kapur K. Do patients bypass rural hospitals? Determinants of inpatient hospital choice in rural California. J Health Care Poor Underserved 2009;20:625-44.

29. Buczko W. Rural Medicare beneficiaries' use of rural and urban hospitals. J Rural Health 2001;17:53-8.

30. Buczko W. Bypassing of local hospitals by rural Medicare beneficiaries. J Rural Health 1994;10: 237-46.

31. Hall MJ, Owings MF. Rural residents who are hospitalized in rural and urban hospitals: United States, 2010. NCHS Data Brief 2014;(159):1-8.

32. Mohr NM, Harland KK, Shane DM, et al. Rural patients with severe sepsis or septic shock who bypass rural hospitals have increased mortality: An instrumental variables approach. Crit Care Med 2017; 45:85-93.

33. Kleinpell R, Vasilevskis EE, Fogg L, Ely EW. Exploring the association of hospice care on patient experience and outcomes of care. BMJ Support Palliat Care 2016 [Epub ahead of print]. 